\title{
Introduction of an electronic referral system in patients with transient ischaemic attacks
}

\author{
Authors: Jemma Shanley and Nigel Smyth
}

\section{Aims}

The primary aim was reduction in time between initial patient assessment and an appointment in the transient ischaemic attack (TIA) clinic. The secondary aim was to improve the quality of data recorded on the referral.

\section{Methods}

Integrated Clinical Environment (ICE), an already established system for requesting imaging and pathology, was adapted to allow for electronic referral and subsequently advertised to existing referrers in primary and secondary care. A database was maintained to allow analyses of this new referral method in comparison with the traditional fax referral. Mean times were calculated to analyse time differences.

\section{Results}

From 25 July 2017 to 17 December 2017 a total of 471 referrals were received (92 ICE and 379 fax referrals). Ninety-two (100\%) of ICE referrals had all data points accurately completed as opposed to $123(32 \%)$ of fax referrals.

The mean time from first assessment to clinic appointment in the primary care patients referred using ICE was 58 hours compared with 110 hours for those referred using fax, a mean difference of 52 hours. The mean time from first assessment to clinic appointment in secondary care patients referred using ICE was 47 hours compared with 77 hours for those referred using fax, a mean difference of 30 hours.

Patients referred from primary care using ICE had a shorter time from initial assessment to their referral being received ( 6 hours vs 30 hours) and a shorter time from the referral being received to an appointment being given ( 52 hours vs 80 hours). Patients referred from secondary care using ICE had a shorter time from initial assessment to their referral received ( 6 hours vs 14 hours) and a shorter time from the referral being received to an appointment being given ( 40 hours vs 66 hours).

The mean time from onset of symptoms to clinic appointment in primary care patients referred using ICE was 95 hours compared with 189 hours for those referred using fax, a mean difference of 94 hours. The mean time from first assessment to clinic appointment in secondary are patients referred using ICE was 66 hours compared with 84 hours, a mean difference of 18 hours.

\section{Conclusion}

Time taken in each step of the patient pathway, from onset of symptoms to initial assessment, receipt of referral and subsequent appointment has been reduced. The improved efficiency seen with ICE referrals is likely down to its automatic receipt by the TIA service compared to the number of individual steps involved in a hand-written fax referral and its subsequent acknowledgement. Creation of an electronic referral form has allowed for the mandatory entry of key diagnostic information.

The net result of this work is that patients with TIA who were referred via ICE experienced improvements in both the speed and accuracy of referral, leading to more timely specialist review which has been demonstrated to improve the overall quality of patient care, potentially reducing hospital bed days, acute costs and 6-month disability.

Further evolution of the care pathway will include a complete switch over to ICE referral after further education of initial assessors in primary and secondary care as well as the provision of enhanced patient information delivery at the time of initial referral. This pathway will also be shared with other local NHS TIA service providers. The ICE referral system could also be potentially used across other services and specialities locally.

\section{Conflict of interest statement}

None declared.

Authors: Stroke Medicine, Royal Hampshire County Hospital, Winchester, UK 